\title{
BMJ Open External validation of the Probability of repeated admission (Pra) risk prediction tool in older community-dwelling people attending general practice: a prospective cohort study
}

\author{
Emma Wallace, ${ }^{1}$ Ronald McDowell, ${ }^{1}$ Kathleen Bennett, ${ }^{2}$ Tom Fahey, ${ }^{1}$ \\ Susan M Smith ${ }^{1}$
}

To cite: Wallace $E$, McDowell R, Bennett $\mathrm{K}$, et al. External validation of the Probability of repeated admission (Pra) risk prediction tool in older community-dwelling people attending general practice: a prospective cohort study. BMJ Open 2016;6:e012336. doi:10.1136/bmjopen-2016012336

- Prepublication history and additional material is available. To view please visit the journal (http://dx.doi.org/ 10.1136/bmjopen-2016012336).

Received 19 April 2016 Revised 7 July 2016 Accepted 8 September 2016

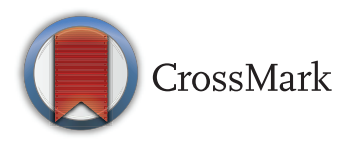

${ }^{1}$ HRB Centre for Primary Care Research, Royal College of Surgeons in Ireland (RCSI), Dublin, Ireland

${ }^{2}$ Population and Health Sciences Division, Royal College of Surgeons in Ireland (RCSI), Dublin, Ireland

Correspondence to Dr Emma Wallace; emmawallace@rcsi.ie

\section{ABSTRACT}

Objectives: Emergency admission is associated with the potential for adverse events in older people and risk prediction models are available to identify those at highest risk of admission. The aim of this study was to externally validate and compare the performance of the Probability of repeated admission (Pra) risk model and a modified version (incorporating a multimorbidity measure) in predicting emergency admission in older community-dwelling people.

Setting: 15 general practices (GPs) in the Republic of Ireland.

Participants: $n=862, \geq 70$ years, community-dwelling people prospectively followed up for 2 years (2010-2012). Exposure: Pra risk model (original and modified) calculated for baseline year where $\geq 0.5$ denoted high risk (patient questionnaire, GP medical record review) of future emergency admission.

Primary outcome: Emergency admission over 1 year (GP medical record review). Statistical analysis: descriptive statistics, model discrimination (c-statistic) and calibration (Hosmer-Lemeshow statistic).

Results: Of 862 patients, a total of $154(18 \%)$ had $\geq 1$ emergency admission(s) in the follow-up year. 63 patients $(7 \%)$ were classified as high risk by the original Pra and of these $26(41 \%)$ were admitted. The modified Pra classified 391 (45\%) patients as high risk and $103(26 \%)$ were subsequently admitted. Both models demonstrated only poor discrimination (original Pra: c-statistic 0.65 (95\% Cl 0.61 to 0.70$)$; modified Pra: c-statistic $0.67(95 \% \mathrm{Cl} 0.62$ to 0.72$)$ ). When categorised according to risk-category model, specificity was highest for the original Pra at cut-point of $\geq 0.5$ denoting high risk (95\%), and for the modified Pra at cut-point of $\geq 0.7(95 \%)$. Both models overestimated the number of admissions across all risk strata.

Conclusions: While the original Pra model demonstrated poor discrimination, model specificity was high and a small number of patients identified as high risk. Future validation studies should examine higher cut-points denoting high risk for the modified Pra, which has practical advantages in terms of

\section{Strengths and limitations of this study}

- This study externally validates the Probability of repeated admissions (Pra) risk model. This tool is easy to use and apply in the general practice (GP) setting but recent external validation is limited.

- GP record data were used to record the medical conditions and healthcare usage questions of the Pra reducing the risk of response bias for these questions.

- This study examines the original Pra and compares its performance to a modified version which substitutes self-rated health and informal carer availability questions (which require patient questionnaire completion) with a measure of multimorbidity. This modified Pra model could be applied using the GP medical record data only in order to risk-stratify patients and has advantages for clinical utility.

- This study was conducted across 15 GPs in Ireland and the findings may not be generalisable to other settings.

application in GP. The original Pra tool may have a role in identifying higher-risk community-dwelling older people for inclusion in future trials aiming to reduce emergency admissions.

\section{BACKGROUND}

Emergency hospital admission in older people is associated with an increased risk of adverse events including falls, functional decline and nosocomial infections. ${ }^{12}$ Recent healthcare policy initiatives in the UK and the USA suggest the use of risk prediction models to identify community-dwelling older people at highest risk of future emergency admission. ${ }^{3}{ }^{4}$ A recent systematic review 
identified 27 such models developed and validated for use in primary care. ${ }^{5}$ One such risk model is the Probability of repeated admission (Pra) tool. ${ }^{6}$ This selfreport questionnaire includes eight items relating to patient demographics (age and gender), specific medical diagnoses (diabetes mellitus and coronary artery disease), healthcare usage (number of doctor visits and emergency admissions in the previous year), self-rated health and the availability of an informal caregiver. Developed in the USA in 1993 for use in older community-dwelling people, the Pra tool is calculated using a logistic formula, with a score of $\geq 0.5$ indicative of high risk of experiencing an emergency admission over the next year. ${ }^{6} 7$

We previously undertook a systematic review and meta-analysis of the Pra tool and identified 10 community-based validation studies and reported a pooled c-statistic of 0.7 , representing poor model discrimination, with high summary specificity (pooled specificity $95 \%$, $95 \%$ CI $95.8 \%$ to $96.7 \%){ }^{8}$ However, the most recent study was conducted over 10 -years ago, so recent external validation is lacking. In addition, the Pra tool, originally designed as a postal questionnaire, has been validated successfully in two US studies using data extracted from health administrative databases and the medical record. ${ }^{9} 10$ These studies excluded Pra questionnaire items relating to self-rated health and caregiver availability and instead substituted a measure of multimorbidity, the Chronic Disease Score and the Deyo-Charlson index, respectively. ${ }^{9}{ }^{10}$ The advantage of this approach is that the modified Pra tool could be applied to a research database or medical record software system without needing the patient to complete a questionnaire, reducing the risk of questionnaire response bias.

The aims of this study were: (1) to externally validate the Pra tool in predicting future emergency admission over 1 year follow-up in a cohort of older communitydwelling people; and (2) to externally validate a modified version of the Pra tool (that substitutes a measure of multimorbidity) in the same study setting and compare predictive accuracy.

\section{METHODS}

The Strengthening The Reporting of Observational Studies in Epidemiology (STROBE) guidelines were adhered to in the conduct and reporting of this cohort study. ${ }^{11}$ In addition, to account for the specific methodological requirements for externally validating a riskprediction model, the Transparent Reporting of a multivariable prediction model for Individual Prognosis or Diagnosis (TRIPOD) statement was adhered to. ${ }^{12}$

\section{Study design and study population}

This is a 2-year prospective cohort study of older general-practice (GP) patients recruited from 15 urban practices in Leinster in the Republic of Ireland (20102012). At baseline in 2010, a proportionate stratified random sampling approach was used to recruit patients for study participation. Study inclusion criteria were: (1) aged $\geq 70$ years on 1 January 2010 , and (2) in receipt of a valid general medical services (GMS) card. Approximately $96 \%$ of all people aged $\geq 70$ years in the Republic of Ireland are in receipt of a GMS card which entitles the holder to free public health services (including GP visits) and prescriptions, subject to a maximum co-payment of $€ 25$ monthly. ${ }^{13}$ The following exclusion criteria were applied: (1) receiving palliative care; (2) cognitive impairment at the level that would impact their ability to complete the outcome measure (defined as Mini Mental State Examination (MMSE) $\leq 20$ ); (3) significant hearing/speech/visual impairment; (4) currently experiencing a psychotic episode; (5) hospitalised long-term, in a nursing home, homeless or in sheltered accommodation; and (6) recent bereavement (within 4 weeks). Each participant's general practitioner applied the exclusion criteria and determined eligibility for participation at baseline in 2010. Ethical approval for this study was granted by the Royal College of Surgeons in Ireland (RCSI) Human Research Ethics committee (reference number REC462bbb) and all participants gave informed consent prior to participating.

\section{Risk prediction tool: calculation of the Pra score}

The Pra score was calculated using postal questionnaire and GP medical record review data collected in 2010. In addition, each patient's GP medical record was reviewed. The original Pra questionnaire items are: (1) age; (2) gender; (3) presence of diabetes mellitus; (4) presence of coronary heart disease; (5) hospital admission in previous year; (6) >6 physician visits in previous year; (7) self-rated health; and (8) availability of an informal caregiver. ${ }^{6}$ A logistic formula is applied to produce a score from 0 to 1 . (See online supplementary 1 ) Patients who score $\geq 0.5$ are considered at high risk of admission over the next year. As the Pra model is under copyright, a no-fee study licence was applied for and granted by the Pra developers to allow use of the Pra logistic formula for the purposes of this study. ${ }^{6}$ The Pra was examined across its three risk categories; low $(<0.35)$, moderate $(0.35-0.49)$ and high $(\geq 0.5)$ as well as an exploratory very-high-risk category of $\geq 0.7$.

In addition, a second modified version of the Pra was calculated. This modified Pra model, validated in two previous studies, substitutes the questionnaire items relating to self-rated health and availability of an informal caregiver for a measure of multimorbidity. ${ }^{9} 10$ The RxRisk-V, a measure developed specifically for older people which classifies patients' chronic medical conditions based on the WHO Anatomical Therapeutic Chemical (WHO-ATC) medication classification system of their dispensed medications, was used as the measure of multimorbidity in this instance. ${ }^{14}$ Therefore, the modified Pra comprised of seven questions; (1) age, (2) gender, (3) presence of diabetes mellitus, (4) presence of coronary heart disease, (5) hospital admission in the previous year, (6) $>6$ physician visits in previous year and 
(7) RxRisk-V score. The Rx-Risk-V was calculated using linked pharmacy claims data from the national Health Services Executive (HSE)-Primary Care Reimbursement Scheme (PCRS) pharmacy claims database. This was available for those who provided consent and who had a valid identifier for linkage purposes. In the HSE-PCRS, dispensed prescriptions are coded using the WHO-ATC classification system, and defined daily doses, strength, quantity of medication and mode of administration are available. This version of the Pra is referred to as modified Pra score and the logistic formula used for calculation is presented in online supplementary material 2. The Pra was examined across three risk categories; low $(<0.35)$, moderate $(0.35-0.49)$ and high $(\geq 0.5)$ as well as an exploratory very-high-risk category of $\geq 0.7$.

The data source used to record each of the Pra questionnaire items (original and modified) is outlined in table 1.

\section{Primary outcome: emergency hospital admission}

Emergency admission was defined as "unplanned overnight stay in hospital'. ${ }^{5}$ This outcome was measured by reviewing the GP medical record over 1 year of follow-up where details regarding emergency admissions (number of admissions, date(s), date of admission(s), length of stay and reason for admission) were recorded for the follow-up year.

\section{Statistical methods}

All descriptive statistics and analysis presented were completed using Stata version-13 (StataCorp, Texas, USA). Two aspects of the Pra model performance were examined in this validation study; discrimination and calibration. Discrimination refers to the ability of the Pra to distinguish correctly the patients with and without the outcome of interest, that is, emergency admission. ${ }^{15}$ The c-statistic with 95\% CIs was estimated using non-parametric receiver operating curve (ROC) analysis with cut points of 0.1 increments. In addition, sensitivity, specificity, positive predictive values (PPVs), negative predictive values (NPVs) and likelihood ratios are presented. Calibration refers to how closely predicted outcomes agree with observed outcomes and therefore is concerned with the number of emergency admissions predicted to occur by the Pra versus the number of emergency admissions actually observed during the prediction period. The Hosmer-Lemeshow goodness of fit statistic was used to estimate the calibration of the Pra score and calibration plots were generated across deciles of risk. ${ }^{16}$

\section{RESULTS}

\section{Baseline characteristics}

Of 904 baseline study participants, a total of 862 (95\%) participants were included in this study. Participants were excluded if there was incomplete hospital admission data for the prediction year. The reasons for exclusion were as follows: 19 moved GP, 14 moved to a nursing home and 9 GP medical record reviews were missing.

The baseline characteristics of the study participants, according to the items in the original and modified Pra models are summarised in table 2.

The proportion of patients at high risk $(\geq 0.5)$ according to the original Pra was $7 \% \quad(n=63)$ compared with $45 \%(\mathrm{n}=391)$ for the modified Pra. This higher proportion was largely due to the higher number of patients with $\geq 5$ RxRisk-V conditions ( $\mathrm{n}=433$ ) using the modified Pra, compared with patients who reported poor or fair health status $(\mathrm{n}=158)$ and lack of an informal caregiver $(n=49)$ applying the original Pra.

\section{Performance of the Pra score: discrimination}

The performance of the two versions of the Pra score in predicting emergency admission over the next year is presented in table 3 .

The c-statistic for the original Pra was 0.65 (95\% CI 0.61 to 0.70 ) compared with 0.67 (95\% CI 0.62 to 0.72 ) for the modified Pra. There was no statistically significant difference in these c-statistics $(p=0.38)$. A total of $41 \%(n=26)$ of patients identified as high risk by the original Pra were subsequently admitted to hospital, while $26 \% \quad(n=103)$ of those stratified as high risk using the modified Pra were subsequently admitted.

Figure 1 presents the ROC curve, a graph of the sensitivity (y-axis) and the 1-specificity (x-axis), for the

Table 1 Data source for each item of the original and modified Pra score

\begin{tabular}{lll}
\hline Pra item & Original Pra score & Modified Pra score \\
\hline Age & GP record & GP record \\
Gender & GP record & GP record \\
Presence of diabetes mellitus & GP record & GP record \\
Presence of coronary heart disease & GP record & GP record \\
Self-rated health & Postal questionnaire & Excluded \\
Formal caregiver availability & Postal questionnaire (proxy of high social & Excluded \\
& support on Lubben's social network scale) & \\
$>6$ physician visits over previous year & GP record & GP record \\
Emergency hospital admission previous year & GP record & GP record \\
Multimorbidity measure: RxRisk-V & Excluded & Linked pharmacy claims database \\
\hline GP, general practice; Pra, Probability of repeated admission. &
\end{tabular}


Table 2 Baseline characteristics of study participants by the original or modified Pra

\begin{tabular}{|c|c|c|}
\hline Patient characteristic & $\begin{array}{l}\text { Original } \\
\text { Pra } \\
(\mathrm{n}=858) \\
\mathrm{N}(\%)\end{array}$ & $\begin{array}{l}\text { Modified } \\
\text { Pra } \\
(\mathrm{n}=862) \\
\mathrm{N}(\%)\end{array}$ \\
\hline Pra score $\geq 0.5$ (high risk) & $63(7)$ & $391(45)$ \\
\hline \multicolumn{3}{|l|}{ Age } \\
\hline $70-74$ & $325(38)$ & \\
\hline $75-79$ & $290(34)$ & \\
\hline $80-84$ & $157(18)$ & \\
\hline$\geq 85$ & $86(10)$ & \\
\hline Coronary artery disease & $148(17)$ & \\
\hline Diabetes mellitus & $101(12)$ & \\
\hline$>6$ doctor visits previous year & $484(56)$ & \\
\hline $\begin{array}{l}\geq 1 \text { emergency admission } \\
\text { previous year }\end{array}$ & $139(16)$ & \\
\hline Poor or fair self-rated health & $158(18)$ & NA \\
\hline No informal caregiver availability* & $49(6)$ & NA \\
\hline \multicolumn{3}{|l|}{ RxRisk-V number of conditions } \\
\hline 0 & NA & $13(2)$ \\
\hline $1-2$ & NA & $156(18)$ \\
\hline $3-4$ & NA & $256(30)$ \\
\hline$\geq 5$ & NA & $433(50)$ \\
\hline
\end{tabular}

*Social support was missing for four study participants.

NA, not applicable; Pra, Probability of repeated admission.

original Pra and the modified Pra, respectively, at incremental Pra score cut-points of 0.1 . The maximum original Pra score in this cohort was 0.71 and the maximum modified Pra score was 0.83 . Both models demonstrated poor model discrimination for the outcome for emergency admission during the 1 year follow-up period. Table 4 presents, at each cut-point, the number of study participants, number admitted, sensitivity, specificity, PPV, NPV and likelihood ratios.

Performance of Pra models at low, moderate, high and very high risk: discrimination

The number of patients per risk category, number admitted, sensitivity, specificity, PPV, NPV and likelihood ratios of the original and modified versions of the Pra at the low, moderate and high-risk cut-off points are presented in table 5 .
The original Pra at a cut-point of $\geq 0.5$ had high specificity of $95 \%$ but low sensitivity of $17 \%$. The NPV for the high-risk category was $84 \%$. The modified Pra reported lower specificity of $59 \%$ at the same cut-point of $\geq 0.5$ but higher sensitivity (67\%). At a 'very-high-risk' category of $\geq 0.7$, the specificity was $95 \%$ with NPV of $84 \%$.

\section{Performance of the Pra score: calibration}

The original and modified versions of the Pra score overestimated the number of hospital admissions in this study population across all levels of risk strata in the 1 year prediction period. The calibration performance of the original and modified Pra models is presented graphically in the calibration plots presented in figures 2 and 3, respectively. The Hosmer-Lemeshow goodness of fit statistic demonstrated a significant difference between observed and predicted admissions for the original Pra $\left(\chi^{2} 84.67, \mathrm{p}\right.$ value $\left.<0.001\right)$ and the modified Pra $\left(\chi^{2}\right.$ $330.23, \mathrm{p}$ value $<0.001)$ indicating overprediction of admissions.

\section{DISCUSSION}

\section{Statement of principal findings}

This validation study compares the predictive accuracy of the original Pra tool and a modified version of the Pra, substituting two self-report items with a measure of multimorbidity, in predicting future hospital admission in older community-dwelling adults.

The original Pra score demonstrated poor discrimination performance (c-statistic 0.63) but identified a relatively small number of people as high risk $(\mathrm{n}=63,7 \%)$, of whom more than one-third $(n=26)$ were admitted to hospital in the outcome year. The modified Pra demonstrated similar poor discrimination performance (c-statistic 0.67) but identified a much higher number of people as high risk $(\mathrm{n}=391,46 \%)$, of whom less than one-third were subsequently admitted $(n=103,26 \%)$. However, adding a 'very-high-risk' category (Pra score $\geq 0.7$ ) for the modified Pra identified only 60 people of whom over one-third $(n=26,43 \%)$ were admitted. Therefore, if this modified model is to be used in future studies then adding an additional risk stratum may be necessary.

Table 3 Prediction of $\geq 1$ emergency admission(s) during 1 year of follow-up by two versions of the Pra score (original and modified)

\begin{tabular}{llc}
\hline & Original Pra $(\mathbf{n = 8 5 8})$ & $\begin{array}{c}\text { Modified Pra }(\mathbf{n}=\mathbf{8 6 2}) \\
\text { c-statistic }(\mathbf{9 5 \%} \mathbf{~ C l})\end{array}$ \\
\hline Prediction of $\geq 1$ emergency admission in follow-up year & c-statistic (95\% Cl) & $0.67(0.62$ to 0.72$)$ \\
& $0.65(0.61$ to 0.70$)$ & $\mathrm{N}(\%)$ \\
Total study participants with $\geq 1$ emergency admission & $\mathrm{N}(\%)$ & $154(18)$ \\
Patients classified as high risk according to Pra score $(\geq 0.5)$ & $154(18)$ & $391(46)$ \\
High-risk patients (Pra score $\geq 0.5)$ with $\geq 1$ emergency & $63(7)$ & $103(26)$ \\
admission (\% of all high risk) & $26(41)$ & \\
Pra, Probability of repeated admission. & &
\end{tabular}




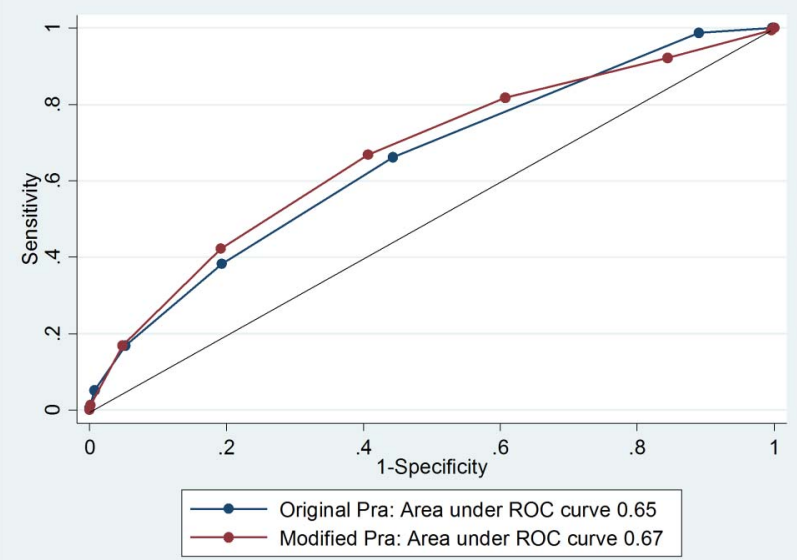

Figure 1 Receiver operating characteristic (ROC) curve for the original and modified probability of repeated admission validation cohort.

Both the original and modified Pra risk models were poorly calibrated in this validation study, with fewer observed emergency admissions compared with predicted admissions across all three strata of risk (low, moderate and high).

\section{Comparison with existing literature}

In the systematic review and meta-analysis of the original Pra described previously, the summary c-statistic was 0.70 with low pooled sensitivity of $12 \%$ (95\% CI $10.5 \%$ to
$13.6 \%)$ and high pooled specificity of $96 \%$ (95\% CI $95.8 \%$ to $96.7 \%){ }^{8}$ The current external validation of the Pra demonstrated poorer model discrimination but the specificity for those classified as high risk was similar in this new setting.

Previous validation studies have been largely conducted in the USA and all were conducted over 10 -years ago. ${ }^{8}$ One of these studies ( $n=6924$ aged $\geq 65$ years), examined the performance of the original Pra in a randomised controlled trial (RCT) across three European countries (UK, Switzerland and Germany). ${ }^{17}$ Similar proportions of patients were classified as high risk, ranging from $4 \%$ to $7 \%$, with similar predictive accuracy in different settings (combined sites c-statistic: 0.64 (95\% CI 0.62 to 0.66)). ${ }^{17}$ Two US community-based studies examined a modified version of the Pra, substituting self-report items (caregiver availability and selfrated health status) for a measure of multimorbidity. One study compared the performance of the original Pra with a modified version (using the Deyo-Charlson index as a multimorbidity measure) in a cohort of older community-dwelling people $(n=296, \geq 65$ years $)$ for the primary outcome of healthcare costs over 6-month follow-up. ${ }^{10}$ This study was limited by a poor response rate to the Pra questionnaire (53\%) but did report similar performance between the two approaches. The specificity of the original Pra at a cut-point of $\geq 0.5$ denoting high risk was $90 \%$ for the outcome of inpatient stay over 6 months, while the modified Pra reported specificity of $92 \%$ for the same outcome. ${ }^{10}$

Table 4 Number of study participants, number admitted, sensitivity, specificity, predictive values and likelihood ratios at cut-points of 0.1 increments for the original and modified Pra

\begin{tabular}{|c|c|c|c|c|c|c|c|c|}
\hline Cut-point & $\begin{array}{l}\text { Number of } \\
\text { participants (n) }\end{array}$ & $\begin{array}{l}\geq 1 \text { emergency } \\
\text { admission (n) }\end{array}$ & $\begin{array}{l}\text { Sensitivity } \\
(\%)\end{array}$ & $\begin{array}{l}\text { Specificity } \\
(\%)\end{array}$ & PPV (\%) & NPV (\%) & LR+ & LR- \\
\hline \multicolumn{9}{|c|}{ Original Pra } \\
\hline$\geq 0$ & 2 & 0 & 100 & 0 & 18 & - & 1.0 & - \\
\hline$\geq 0.1$ & 77 & 2 & 100 & 0 & 18 & 100 & 1.0 & 0.0 \\
\hline$\geq 0.2$ & 365 & 50 & 99 & 11 & 20 & 97 & 1.1 & 0.1 \\
\hline$\geq 0.3$ & 219 & 43 & 66 & 56 & 25 & 88 & 1.5 & 0.6 \\
\hline$\geq 0.4$ & 132 & 33 & 38 & 81 & 30 & 86 & 2.0 & 0.8 \\
\hline$\geq 0.5$ & 50 & 18 & 17 & 95 & 41 & 84 & 3.2 & 0.9 \\
\hline$\geq 0.6$ & 12 & 7 & 5 & 99 & 62 & 83 & 7.3 & 1.0 \\
\hline$\geq 0.7$ & 1 & 1 & 1 & 100 & 100 & 82 & - & 1.0 \\
\hline$\geq 0.8$ & 0 & 0 & 0 & 100 & - & 82 & - & 1.0 \\
\hline$\geq 0.9$ & 0 & 0 & 0 & 100 & - & 82 & - & 1.0 \\
\hline \multicolumn{9}{|c|}{ Modified Pra } \\
\hline$\geq 0$ & 0 & 0 & 100 & 0 & 18 & - & 1.0 & - \\
\hline$\geq 0.1$ & 4 & 1 & 100 & 0 & 18 & - & 1.0 & - \\
\hline$\geq 0.2$ & 118 & 11 & 99 & 0 & 18 & 75 & 1.0 & 1.5 \\
\hline$\geq 0.3$ & 184 & 16 & 81 & 16 & 19 & 90 & 1.1 & 0.5 \\
\hline$\geq 0.4$ & 165 & 23 & 81 & 39 & 49 & 90 & 1.3 & 0.5 \\
\hline$\geq 0.5$ & 190 & 38 & 67 & 59 & 26 & 89 & 1.6 & 0.6 \\
\hline$\geq 0.6$ & 141 & 39 & 42 & 81 & 32 & 87 & 2.2 & 0.7 \\
\hline$\geq 0.7$ & 57 & 24 & 17 & 95 & 43 & 84 & 3.5 & 0.9 \\
\hline$\geq 0.8$ & 3 & 2 & 1 & 100 & 67 & 82 & 9.2 & 1.0 \\
\hline$\geq 0.9$ & 0 & 0 & 0 & 100 & - & 82 & - & 1.0 \\
\hline
\end{tabular}


Table 5 Number of study participants, number admitted, sensitivity, specificity, predictive values and likelihood ratios at low, moderate, high and very-high-risk categories for the original Pra and modified Pra

\begin{tabular}{|c|c|c|c|c|c|c|c|c|}
\hline Risk category & $\begin{array}{l}\text { Number of } \\
\text { participants }(n)\end{array}$ & $\begin{array}{l}\geq 1 \text { emergency } \\
\text { admission ( } n)\end{array}$ & $\begin{array}{l}\text { Sensitivity } \\
(\%)\end{array}$ & $\begin{array}{l}\text { Specificity } \\
(\%)\end{array}$ & $\begin{array}{l}\text { PPV } \\
(\%)\end{array}$ & $\begin{array}{l}\text { NPV } \\
(\%)\end{array}$ & LR+ & LR- \\
\hline \multicolumn{9}{|l|}{ Original Pra } \\
\hline Low risk (0-0.34) & 569 & 73 & 100 & 0 & 18 & - & 1.0 & - \\
\hline Moderate risk $(0.35-0.49)$ & 226 & 55 & 53 & 70 & 28 & 87 & 1.7 & 0.7 \\
\hline High risk (0.5-0.69) & 62 & 25 & 17 & 95 & 41 & 84 & 3.2 & 0.9 \\
\hline Very high risk $(\geq 0.7)$ & 1 & 1 & 1 & 100 & 100 & 82 & - & 1.0 \\
\hline \multicolumn{9}{|l|}{ Modified Pra } \\
\hline Low risk (0-0.34) & 198 & 18 & 100 & 0 & 18 & - & 1.0 & - \\
\hline Moderate risk $(0.35-0.49)$ & 273 & 33 & 88 & 25 & 20 & 91 & 1.1 & 0.5 \\
\hline High risk (0.5-0.69) & 331 & 77 & 67 & 59 & 26 & 89 & 1.6 & 0.6 \\
\hline Very high risk $(\geq 0.7)$ & 60 & 26 & 17 & 95 & 43 & 84 & 3.5 & 0.9 \\
\hline
\end{tabular}

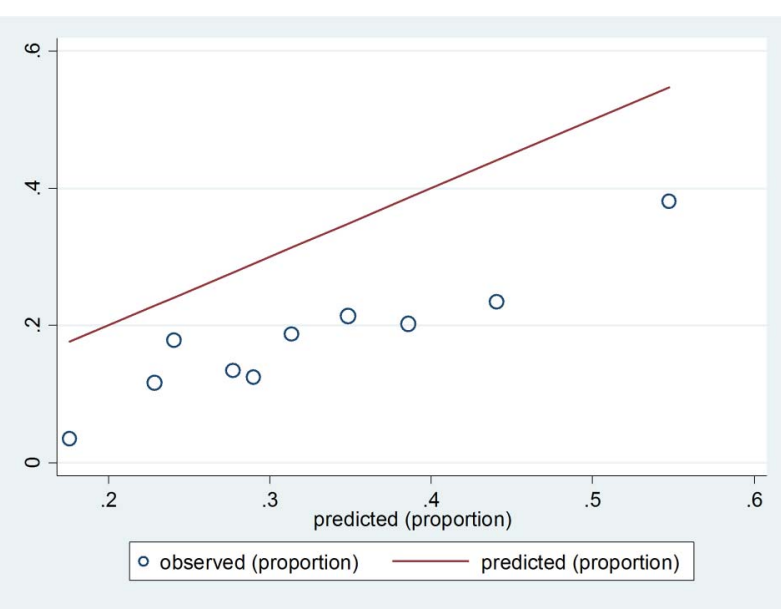

Figure 2 Calibration performance in deciles of risk for the original probability of repeated admission score.

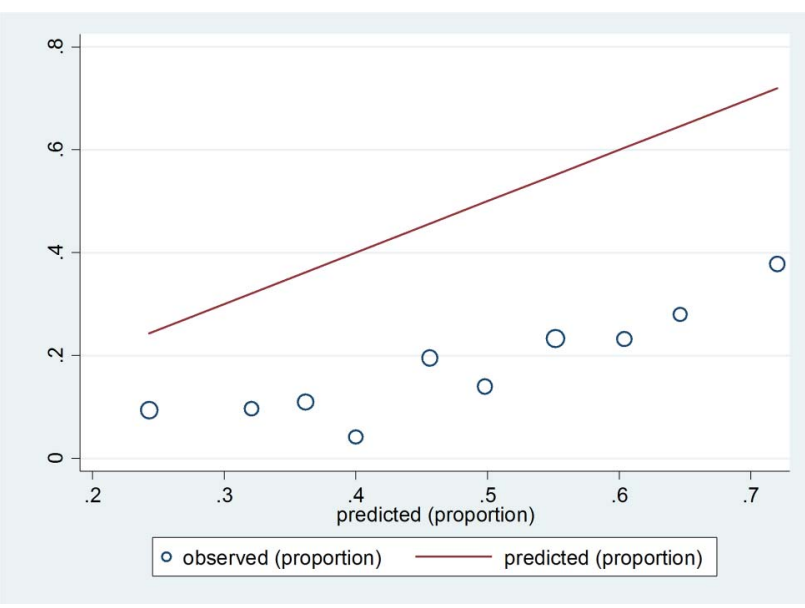

Figure 3 Calibration performance in deciles of risk for the modified probability of repeated admission score.

A second study of US older community-dwelling people $(n=2174$, aged $\geq 65$ years $)$ used the Chronic Disease Score as a surrogate for self-reported health status and caregiver availability. ${ }^{9}$ This study reported similar predictive accuracy using either model (original Pra c-statistic: 0.69, modified Pra c-statistic: 0.69) and similar proportions of patients identified as high risk (14\% and $16 \%$, respectively, for original and modified Pra). ${ }^{9}$ However, as this study was published in 1998 and was based on data from one USA state, the generalisability of its findings to current practice and other healthcare settings is somewhat limited.

The Pra tool performs similarly to other models based on self-report and designed for use in primary care. ${ }^{5}$ Examples include the Sherbrooke questionnaire and the Emergency Admission Risk Likelihood Index (EARLI), which include similar items relating to previous healthcare use, self-rated health and medical diagnoses with similar predictive accuracy. ${ }^{18} 19$ In external validation studies, the Sherbrooke questionnaire reported poor model discrimination (c-statistic: 0.60 (95\% CI 0.53 to 0.67)) similar to the EARLI with reported c-statistic of 0.67 (95\% CI 0.63 to 0.70$).{ }^{18}{ }^{19}$ Other risk prediction models designed using routine data sets or clinical record review data tend to include a much larger numbers of variables and often require the availability of linked data sets (eg, inpatient/OPD/primary care) for application. ${ }^{5}$ For example, the UK QAdmissions model was developed using linked computerised GP and hospital inpatient data and includes 30 variables (c-statistic 0.77 (95\% CI 0.771 to 0.774$).{ }^{20}$ The predictive accuracy of these types of models tends to be higher overall but their application depends largely on the availability of large linked data sets. In many countries, including Ireland, there is no data set available that links primary care and hospital usage data, so applying a model, such as the QAdmissions, for risk-stratification purposes would be very challenging.

Regarding calibration performance, the Pra models overpredicted admissions across all risk categories in the current study. Case mix variation is a particular issue for external validation studies with heterogeneity of predictor variables and outcomes of interest across study populations. ${ }^{21}$ Differences in the prevalence of the outcome of interest, in this case emergency admission, 
can impact on the performance of the model. This is one reason for updating the original risk model where the intercept is updated to recalibrate predictive performance for a new setting. ${ }^{21}$

\section{Clinical and research implications}

Recent policy initiatives internationally suggest the application of community-initiated risk stratification to identify higher-risk people who can be prioritised for an intervention to reduce their risk of future emergency admission. ${ }^{3}$ However, this approach requires an accurate admission risk-stratification tool as well as effective community-based interventions and current evidence for both is limited. ${ }^{22}$

The Pra tool has been used as a risk-stratification tool in two studies examining the effectiveness of communitybased interventions in improving different patient outcomes including reduced healthcare usage. ${ }^{23}{ }^{24}$ In one of these RCTs targeting older people at increased risk of functional decline, the Pra was used to identify higherrisk people who were then prioritised for an intervention. ${ }^{23}$ This complex intervention included the following components: (1) training of health professionals; (2) administration of the Health Risk Appraisal for Older persons questionnaire; (3) group education sessions with additional personal reinforcement; (4) home visits by a trained nurse with detailed feedback and discussion with the geriatric team; and (5) written feedback to the participant's general practitioner. ${ }^{23}$ This intervention had moderately favourable effects on the primary outcomes of preventative care use (uptake of vaccinations eg, influenza vaccinations, adjusted OR 1.7, (95\% CI 1.4 to 2.1) and more favourable health behaviour (physical activity and diet, eg, high fruit/fibre intake, OR 2.0, (95\% CI 1.6 to 2.6), as compared with controls. ${ }^{23}$

The Pra has also been used in a similar context to identify high-risk patients for inclusion in another RCT measuring the effectiveness of an outpatient geriatric evaluation and management plan on functional decline. ${ }^{24}$ Patients in the intervention group were significantly less likely than the control group to lose functional ability (adjusted OR 0.67 , (95\% CI 0.47 to 0.99 )) at 18-month follow-up. However, other outcomes such as mortality, use of most health services (with the exception of home health services), and total healthcare costs did not differ significantly between the two groups. ${ }^{24}$

Based on the findings of the current study, use of the original Pra at cut-point of $\geq 0.5$ may have a role in identifying higher-risk patients for enrolment in RCTs in community settings, as it identified a much smaller number of patients as high risk and demonstrated similar predictive accuracy and better calibration than the modified Pra score at this cut-point. However, at a higher cut-point of $\geq 0.7$, the modified Pra demonstrated similar predictive accuracy and has the advantage of applicability to the GP record rather than relying on patient questionnaire data, so could be explored in future validation studies at this cut-point. The NPV of the Pra is quite high $(\geq 84 \%)$ for patients risk-stratified as moderate or high risk and therefore can be useful as a screening test in determining which patients in the community would not benefit from an intervention aiming to reduce future emergency admission rate. However, its PPV is low ( $40 \%$ for high-risk group or very-high-risk group) indicating that a significant proportion of patients who are stratified as high risk will not go on to experience an emergency admission.

\section{Study limitations}

The items of the Pra score relating to presence of diabetes, coronary artery disease and previous healthcare use were recorded from the GP record rather than the patient questionnaire as per the original Pra. However, it may be argued that this is a more accurate representation of these items as self-report is more prone to response bias. In addition, the outcome of interest, emergency admission in the follow-up year was also recorded from the GP medical record. Previous studies have used different approaches in ascertaining future admission including survey and medical record review. This study recruited patients from 15 urban GP practices in Leinster in the Republic of Ireland and excluded patients with moderate or severe cognitive impairment which may reduce the generalisability of the findings. However, the Pra model was designed as a patient questionnaire which relies on the respondent's ability to recall, among other items, doctor visits and emergency admission. As such, it was not designed for use in a cognitively impaired population. The modified Pra may have a role in this population but this needs to be tested in future validation studies.

\section{Conclusions}

The original Pra tool demonstrated poor discrimination but high specificity in this external validation study and identified a relatively small proportion of patients as high risk. This study suggests that the modified Pra tool, incorporating a multimorbidity measure, is more useful at a higher cut-point indicating high risk which could be examined in future validation studies. The Pra tool may have a role in identifying higher-risk older people for inclusion in future trials aiming to improve outcomes for older community-dwelling people.

Acknowledgements We thank Dr Caitriona Cahir who led the baseline study data collection and the Health Services Executive (HSE)-Primary Care Reimbursement Scheme (PCRS) for use of pharmacy claims database.

Contributors EW led the study, did the statistical analysis and wrote the paper. $\mathrm{RMcD}$ offered methodological guidance for the statistical analysis and contributed to the paper. KB offered significant methodological guidance to the planning of the study, the data analysis and contributed to the final paper. TF provided clinical and methodological guidance, and contributed to the final paper. SMS provided clinical and methodological guidance and contributed to the final paper.

Funding EW is funded by the Health Research Board (HRB) of Ireland under the Research Training Fellowship for Healthcare Professionals award, grant 
no. HPF/2012/20. This research was conducted as part of the HRB Scholar's programme in Health Services Research under grant no. $\mathrm{PhD} / 2007 / 16$ at the HRB Centre for Primary Care Research, grant HRC/2007/1.

Competing interests None declared.

Patient consent Obtained.

Ethics approval Research Ethics Committee at the Royal College of Surgeons in Ireland.

Provenance and peer review Not commissioned; externally peer reviewed.

Data sharing statement No additional data are available.

Open Access This is an Open Access article distributed in accordance with the Creative Commons Attribution Non Commercial (CC BY-NC 4.0) license, which permits others to distribute, remix, adapt, build upon this work noncommercially, and license their derivative works on different terms, provided the original work is properly cited and the use is non-commercial. See: http:// creativecommons.org/licenses/by-nc/4.0/

\section{REFERENCES}

1. de Vries EN, Ramrattan MA, Smorenburg SM, et al. The incidence and nature of in-hospital adverse events: a systematic review. Qual Saf Health Care 2008;17:216-23.

2. Thomas EJ, Brennan TA. Incidence and types of preventable adverse events in elderly patients: population based review of medical records. BMJ 2000;320:741-4.

3. Avoiding unplanned admissions enhanced service: proactive case finding and care review for vulnerable people. Guidance and audit requirements. June 2014, NHS England. https://www.england.nhs.uk/ commissioning/wp-content/uploads/sites/12/2016/04/aua-serv-spec.pdf

4. Summary of final rule provisions for Accountable Care Organizations under the Medicare Shared Savings Program. April 2014. Centers for Medicare and Medicaid services. http://www.cms.gov/medicare/ medicare-fee-for-service-payment/sharedsavingsprogram/ downloads/aco summary factsheet icn907404.pdf (accessed on 5 Dec 2014).

5. Wallace E, Stuart E, Vaughan N, et al. Risk prediction models to predict emergency hospital admission in community-dwelling adults: a systematic review. Med Care 2014;52:751-65.

6. Boult C, Dowd B, McCaffrey D, et al. Screening elders for risk of hospital admission. J Am Geriatr Soc 1993;41:811-17.

7. Pacala JT, Boult C, Boult L. Predictive validity of a questionnaire that identifies older persons at risk for hospital admission. J Am Geriatr Soc 1995;43:374-7.

8. Wallace $\mathrm{E}$, Hinchey $\mathrm{T}$, Dimitrov BD, et al. A systematic review of the probability of repeated admission score in community-dwelling adults. J Am Geriatr Soc 2013;61:357-64.
9. Coleman EA, Wagner EH, Grothaus LC, et al. Predicting hospitalization and functional decline in older health plan enrollees: are administrative data as accurate as self-report? J Am Geriatr Soc 1998;46:419-25.

10. Vojta CL, Vojta DD, Tenhave TR, et al. Risk screening in a Medicare/Medicaid population administrative data versus self report. $J$ Gen Intern Med 2001;16:525-30.

11. von Elm E, Altman DG, Egger M, et al. The Strengthening the Reporting of Observational Studies in Epidemiology (STROBE) statement: guidelines for reporting observational studies. $J$ Clin Epidemiol 2008;61:344-9.

12. Moons KG, Altman DG, Reitsma JB, et al. New guideline for the reporting of studies developing, validating, or updating a multivariable clinical prediction model: the TRIPOD Statement. Adv Anat Pathol 2015;22:303-5.

13. Health Service Executive. Primary care reimbursement service statistical analysis of claims and payments 2011. Dublin: HSE, 2013.

14. Fishman PA, Goodman MJ, Hornbrook MC, et al. Risk adjustment using automated ambulatory pharmacy data: the RxRisk model. Med Care 2003;41:84-99.

15. Swets J. Measuring the accuracy of diagnostic systems. Science 1998;240:1285-93.

16. Cook NR. Use and misuse of the receiver operating characteristic curve in risk prediction. Circulation 2007;115:928-35.

17. Wagner JT, Bachmann LM, Boult C, et al. Predicting the risk of hospital admission in older persons-Validation of a brief self-administered questionnaire in three European countries. J Am Geriatr Soc 2006;54:1271-6.

18. Daniels $R$, van Rossum E, Beurskens A, et al. The predictive validity of three self-report screening instruments for identifying frail older people in the community. BMC Public Health 2012;12:69.

19. Lyon D, Lancaster GA, Taylor S, et al. Predicting the likelihood of emergency admission to hospital of older people: development and validation of the Emergency Admission Risk Likelihood Index (EARLI). Fam Pract 2007;24:158-67.

20. Hippisley-Cox J, Coupland C. Predicting risk of emergency admission to hospital using primary care data: derivation and validation of QAdmissions score. BMJ Open 2013;3:e003482.

21. Riley RD, Ensor J, Snell KI, et al. External validation of clinica prediction models using big datasets from e-health records or IPD meta-analysis: opportunities and challenges. BMJ 2016;353:i3140.

22. Wallace $\mathrm{E}$, Smith SM, Fahey $\mathrm{T}$, et al. Reducing emergency admissions through community based interventions. BMJ 2016;352: h6817.

23. Stuck AE, Kharicha K, Dapp U, et al. The PRO-AGE study: an international randomised controlled study of health risk appraisal for older persons based in general practice. BMC Med Res Methodol 2007;7:2.

24. Boult C, Boult LB, Morishita L, et al. A randomized clinical trial of outpatient geriatric evaluation and management. J Am Geriatr Soc 2001;49:351-9. 